\title{
Assessment of the value of serum cholinesterase as a liver function test for cirrhotic patients
}

\author{
FANPING MENG $^{1 *}$, XIAOJUAN YIN ${ }^{2 *}$, XUEMEI MA ${ }^{1}$, XIAO-DONG GUO ${ }^{3}$, BO JIN $^{1}$ and HANWEI LI ${ }^{1}$ \\ ${ }^{1}$ Liver Cirrhosis Diagnosis and Therapy Center, 302 Hospital of PLA, Beijing 100039; \\ ${ }^{2}$ Affiliated Bayi Children's Hospital, Beijing Military Region General Hospital, Beijing 100700; \\ ${ }^{3}$ Pathological Diagnosis and Research Center, 302 Hospital of PLA, Beijing 100039, P.R. China
}

Received October 21, 2012; Accepted January 3, 2013

DOI: $10.3892 /$ br.2013.60

\begin{abstract}
A variety of laboratory tests are used in the evaluation of hepatic patients. Serum cholinesterase is reduced in liver dysfunction in contrast to other enzymes. The aim of this study was to assess the value of serum cholinesterase in evaluating liver reserve function in cirrhotic patients. A total of 866 cirrhotic patients were divided into three groups according to their Child-Pugh score. Serum cholinesterase of the patients was detected using the enzyme rate method. Simultaneously, serum albumin was detected using the bromocresol green test, while serum plasma prothrombin time was detected using the clotting assay. Using analysis of variance, the value of serum cholinesterase was analyzed in evaluating the liver reserve function of cirrhotic patients in different Child grades. Using correlation analysis, the correction between cholinesterase and albumin and serum plasma prothrombin time was analyzed. Cirrhotic patients were grouped strictly into A, B and C grades, as per the Child-Pugh score. The results showed that cholinesterase tended to significantly decrease in the three grades Child A (5368.04 \pm 1657.32 U/1), Child B (2943.06 \pm 1212.84 U/1) and Child C (1832.51 $\pm 710.68 \mathrm{U} / \mathrm{l})(\mathrm{F}=264.135, \mathrm{P}=0.000)$. In patients with cirrhosis, cholinesterase was positively correlated with albumin and negatively correlated with serum plasma prothrombin time. In the Child A grade, serum cholinesterase was positively correlated with albumin, but negatively correlated with serum plasma prothrombin time. In the Child B grade, serum cholinesterase remained negatively correlated with serum plasma prothrombin time although there was no
\end{abstract}

Correspondence to: Dr Hanwei Li, Liver Cirrhosis Diagnosis and Therapy Center, 302 Hospital of PLA, Xi-si-huan-zhong Road 100, Beijing 10039, P.R. China

E-mail: drlihanwei@gmail.com

${ }^{*}$ Contributed equally

Abbreviations: LFTs, liver function tests; PT, prothrombin time

Key words: serum cholinesterase, liver cirrhosis, Child-Pugh score, albumin, prothrombin time significant correlation between cholinesterase and albumin. In the Child $\mathrm{C}$ grade, serum cholinesterase positively correlated with albumin, but there was no significant correlation between cholinesterase and serum plasma prothrombin time. In conclusion, correlated with the damage severity of liver cells, cholinesterase may respond to liver reserve function.

\section{Introduction}

Laboratory tests, often known as liver function tests (LFTs), are useful in the evaluation and treatment of patients with hepatic dysfunction, comprising serum aspartate and alanine transaminases, alkaline phosphatase, bilirubin and albumin. These tests often reveal abnormal results in patients with clinical problems other than liver dysfunction (1). Tests of the biosynthetic capacity of the liver include serum albumin, ceruloplasmin, ferritin, $\alpha 1$-antitrypsin, lipoproteins and bloodclotting factors. These substances are synthesized in the liver for transport into the circulation. Cholinesterase is synthesized mainly in hepatocytes and is released into the blood (2). Serum cholinesterase activity is reduced in liver dysfunction due to reduced synthesis. This is in contrast to other serum enzymes associated with the clinical assessment of liver function whose activities increase as a result of enhanced release from their cellular sources following cell membrane damage (3).

In gastroenterology, the Child-Pugh score (also known as the Child-Turcotte-Pugh score) is used to assess the prognosis of chronic liver disease, mainly cirrhosis. Although originally used to predict mortality during surgery, the Child-Pugh score is now used to determine the prognosis as well as the required strength of treatment, and the necessity for liver transplantation. As two main clinical measures in Child-Pugh score, serum protein and blood-clotting factors are important in evaluating the liver reserve function of cirrhotic patients (4). However, the cirrhotic patients, particularly those with Child grades $\mathrm{B}$ and $\mathrm{C}$ with ascites or hemorrhagic tendency, are usually treated with albumin or blood transfusion, which may affect the real numerical value for calculating the Child-Pugh score (5). Additionally, serum cholinesterase is not easily affected by this treatment. We therefore compared serum cholinesterase with the Child-Pugh score to evaluate the liver reserve function of cirrhotic patients. Moreover, since serum cholinesterase, albumin and blood-clotting factors are synthesized 
by the liver with different half-life (6), we also monitored the correction between cholinesterase, albumin and serum plasma prothrombin time.

\section{Patients and methods}

Patients. In total, 866 cirrhotic patients presenting at the 302 Hospital of the People's Liberation Army (Beijing, China) between January, 2008 and January, 2012 were included in the present study. Inclusion criteria (7) for the study were: a diagnosis of liver cirrhosis based on histology, clinical or ultrasonographic signs or on transient elastography (FibroScan; Echosens, Paris, France). Exclusion criteria were: i) history of albumin or blood transfusion four weeks prior to enrolment; ii) history or clinical evidence of variceal bleeding at enrolment; iii) history or evidence of hepatocellular carcinoma on the basis of ultrasonography, $\alpha$-fetoprotein levels $(<400 \mathrm{lg} / \mathrm{l})$ and/or histology at entry; and iv) liver transplantation. Informed consent was obtained from patients included in the study.

Measurement of biochemical serum markers. Blood samples were collected with minimal venostasis. Serum was obtained from clotted blood by centrifugation within $1 \mathrm{~h}$ of sampling. LFTs comprising serum albumin and prothrombin time were carried out. Serum cholinesterase activity was determined using an assay with butyrylthiocholine as the substrate. The analyses were carried out within $2 \mathrm{~h}$ of sample separation using Boehringer reagent kits (Boehringer Mannheim GmbH, Mannheim, Germany) on an Olympus AU5400 Automatic Analyzer (Olympus Ltd., Tokyo, Japan.)

Statistical analysis. Database management and the statistical analysis were performed using the SPSS software for Windows (ver. 17.0; SPSS, Chicago, IL, USA). Descriptive results were expressed as the mean \pm standard deviation (SD) or number (percentage) of patients with a condition. Multiple comparisons for the ANOVA test were used to compare the mean data. The Pearson correction test was applied for the comparison of cholinesterase, albumin and serum prothrombin times. The tests were two-tailed and $\mathrm{P}<0.05$ was considered to indicate a statistically significant difference.

\section{Results}

Patient characteristics. A total of 681 male and 185 female patients (mean age, 53.2 \pm 11.2 years) were included. The main demographic, laboratory and endoscopic features of the patients are summarized in Table I. The Child A group was present in $437(50.5 \%)$ patients, while the Child B and C groups were evident in $283(32.7 \%)$ and $146(16.9 \%)$ patients, respectively.

Serum cholinesterase in various Child-Pugh score groups. The cirrhotic patients were strictly grouped into the A, B and $\mathrm{C}$ groups, based on their Child-Pugh score. The results showed that serum cholinesterase tended to decrease significantly in the three grades: Child A (5368.04 $\pm 1657.32 \mathrm{U} / \mathrm{l})$, Child B $(2943.06 \pm 1212.84 \mathrm{U} / \mathrm{l})$ and Child C (1832.51 $\pm 710.68 \mathrm{U} / \mathrm{l})$ $(\mathrm{F}=264.135, \mathrm{P}=0.000)$ (Table II). Difference between the mean serum cholinesterase activity in the Child A, B and C groups
Table I Clinical and demographic characteristics of the study population.

\begin{tabular}{lc}
\hline Characteristics & $\mathrm{n}=866$ \\
\hline Age (years) & \\
Mean \pm SD & $53.2 \pm 11.2$ \\
Range & $22-66$ \\
Male gender, $\mathrm{n}(\%)$ & $681(78.6 \%)$ \\
Bilirubin $(\mu$ mol/l) & $30.2 \pm 25.4$ \\
AST (IU/l) & $87.3 \pm 63.5$ \\
ALT (IU/l) & $68.5 \pm 58.4$ \\
Albumin $(\mathrm{g} / \mathrm{l})$ & $31.6 \pm 6.2$ \\
PT (sec) & $14.4 \pm 2.2$ \\
Serum cholinesterase & $3687.7 \pm 1443.2$ \\
Child-Pugh class (scores) & \\
A (5-6) & $437(50.5 \%)$ \\
B (7-9) & $283(32.7 \%)$ \\
C (10-15) & $146(16.9 \%)$ \\
\hline
\end{tabular}

Values are mean \pm standard deviation (SD) or $\mathrm{n}(\%)$. AST, aspartate aminotransferase; ALT, alanine aminotransferase; PT, prothrombin time.

Table II. Serum cholinesterase in the Child-Pugh groups of cirrhotic patients(U/1, mean $\pm \mathrm{SD})$.

\begin{tabular}{lcl}
\hline Group & Cases & Cholinesterase \\
\hline Child A & 437 & $5368.04 \pm 1657.32$ \\
Child B & 283 & $2943.06 \pm 1212.84$ \\
Child C & 146 & $1832.51 \pm 710.68$ \\
\hline
\end{tabular}

Difference between the groups by one-way ANOVA $(\mathrm{F}=264.135$, $\mathrm{P}=0.000$ ). Multiple comparisons with the Child-Pugh A, B, C groups using lysergic acid diethylamide (LSD) $\left(\mathrm{P}_{\mathrm{AB}}=0.000 ; \mathrm{P}_{\mathrm{AC}}=0.000\right.$, $\mathrm{PBC}=0.000)$.

Table III. Correlation between cholinesterase, albumin and plasma prothrombin time in cirrhotic patients.

\begin{tabular}{lcc}
\hline Related pairs & Correlation index & P-value \\
\hline CHE: ALB & $r=0.633$ & 0.000 \\
CHE: PTs & $r=-0.571$ & 0.000 \\
ALB: PTs & $r=-0.466$ & 0.000 \\
\hline
\end{tabular}

CHE, cholinesterase; ALB, albumin; PT, prothrombin time.

was statistically significant, as was the difference between the mean values for the Child B and $\mathrm{C}$ groups.

Correlation between serum cholinesterase, albumin and plasma prothrombin time. Cholinesterase was positively 
Table IV. Correlation between cholinesterase, albumin and plasma prothrombin time in the Child A group of cirrhotic patients.

\begin{tabular}{lcc}
\hline Related pairs & Correlation index & P-value \\
\hline CHE: ALB & $\mathrm{r}=0.413$ & 0.000 \\
CHE: PTs & $\mathrm{r}=-0.456$ & 0.000 \\
ALB: PTs & $\mathrm{r}=-0.258$ & 0.000 \\
\hline
\end{tabular}

CHE, cholinesterase; ALB, albumin; PT, prothrombin time.

Table V. Correlation between cholinesterase, albumin and plasma prothrombin time in the Child B group of cirrhotic patients.

\begin{tabular}{lcc}
\hline Related pairs & Correlation index & P-value \\
\hline CHE: ALB & $\mathrm{r}=0.018$ & 0.828 \\
CHE: PTs & $\mathrm{r}=-0.186$ & 0.015 \\
ALB: PTs & $\mathrm{r}=-0.184$ & 0.016 \\
\hline
\end{tabular}

CHE, cholinesterase; ALB, albumin; PT, prothrombin time.

Table VI. Correlation between cholinesterase, albumin and plasma prothrombin time in the Child $\mathrm{C}$ group of cirrhotic patients.

\begin{tabular}{lcc}
\hline Related pairs & Correlation index & P-value \\
\hline CHE: ALB & $r=0.430$ & 0.000 \\
CHE: PTs & $r=-0.153$ & 0.176 \\
ALB: PTs & $r=-0.186$ & 0.096 \\
\hline
\end{tabular}

CHE, cholinesterase; ALB, albumin; PT, prothrombin time.

correlated with albumin $(\mathrm{r}=0.633, \mathrm{P}=0.000)$ and negatively correlated with plasma prothrombin time $(\mathrm{r}=-0.571, \mathrm{P}=0.000)$ in the cirrhotic patients, confirming that those substances were synthesized in the liver and reduced in liver dysfunction, due to reduced synthesis. In the Child A group, serum cholinesterase was also positively correlated with albumin $(\mathrm{r}=0.413, \mathrm{P}=0.000)$ and negatively correlated with plasma prothrombin time $(\mathrm{r}=-0.456, \mathrm{P}=0.000)$. In the Child B group, cholinesterase remained negatively correlated with plasma prothrombin time $(\mathrm{r}=-0.186, \mathrm{P}=0.015)$, but there was no significant correlation with albumin $(\mathrm{r}=0.018, \mathrm{P}=0.828)$. In the Child $\mathrm{C}$ group, cholinesterase positively correlated with albumin $(r=0.430, P=0.000)$, but there was no significant correlation with plasma prothrombin time ( $\mathrm{r}=-0.153$, $\mathrm{P}=0.176$ ). However, albumin was negatively correlated with plasma prothrombin time in the Child A group of cirrhotic patients although no significant correlation was detected in the Child C group (Tables III-VI).

\section{Discussion}

In biochemistry, cholinesterase is a family of enzymes that catalyzes the hydrolysis of the neurotransmitter acetylcholine into choline and acetic acid, a reaction necessary to allow a cholinergic neuron to return to its resting state after activation (8). There are two types of cholinesterases acetylcholinesterase (AchE), also known as RBC and erythrocyte cholinesterases or acetylcholine acetylhydrolase, found primarily in the blood and neural synapses. AchE exists in various molecular forms. In the mammalian brain the majority of AchE occurs as a tetrameric, G4 form with much smaller amounts of a monomeric G1 form (9). Pseudocholinesterase, also termed plasma cholinesterase, butyrylcholinesterase or acylcholine acylhydrolase, is found primarily in the liver $(10,11)$.

Estimation of the level of activity of the cholinesterase found in serum was first suggested by McArdle (1940) (12), as a useful means for differentiating hepatic from post-hepatic jaundice. The evidence which has accumulated suggests that cholinesterase activity is an assessment indicator for liver function in patients with liver disease. In China, cholinesterase has been included in scores to distinguish hepatitis severity by society of liver disease (7). However, few studies are available regarding the value of cholinesterase in evaluating liver reserve function of cirrhotic patients. The Child-TurcottePugh scoring system is the first of its kind in stratifying the seriousness of end-stage liver disease, mainly cirrhosis (13). In our study, 866 cirrhotic patients were strictly grouped into A, B and C grades, as per their Child-Pugh score. The results show that cholinesterase tended to significantly decrease in the three grades: Child A $(5368.04 \pm 1657.32 \mathrm{U} / 1)$, Child B $(2943.06 \pm 1212.84 \mathrm{U} / \mathrm{l})$ and Child C $(1832.51 \pm 710.68 \mathrm{U} / \mathrm{l})$. This result is in agreement with the findings of Gu and Zhong (14). Their data demonstrated that cholinesterase in the three grades were: Child A $(5978 \pm 535 \mathrm{U} / \mathrm{l})$, Child B $(3957 \pm 454 \mathrm{U} / \mathrm{l})$ and Child C (2267 \pm 332 U/1). The Child-Pugh score employs five clinical measures of liver disease, among which ascites and hepatic encephalopathy are subjective measures (15). Liver cirrhosis is classified into Child-Pugh class A-C, employing the added score from above. Compared with the Child-Pugh score, cholinesterase is easier and more objective in evaluating the liver reserve function of cirrhotic patients.

The liver performs an important role in biosynthesis. Cholinesterase, albumin and blood-clotting factors are synthesized in the liver to be transported into the circulation. Thus, LFTs include cholinesterase, albumin and prothrombin time and may provide useful information concerning the state of a cirrhotic patient's liver. In our study, in cirrhotic patients, cholinesterase was positively correlated with albumin and negatively correlated with plasma prothrombin time, confirming that those substances were synthesized in the liver and reduced in liver dysfunction due to reduced synthesis. In decompensated cirrhotic patients, albumin or blood transfusion are usually used, which may affect the real numerical value to calculate the Child-Pugh score (16). If surgery is selected as the method of treatment based on the effected Child-Pugh scores, the risk for liver failure is likely to increase (17). In their study, Gu and Zhong (14) demonstrated that three cirrhotic patients (two Child B- and one Child A-score patient) suffered hepatic 
encephalopathy following portal azygous disconnection operation, with cholinesterase levels of $<2,000 \mathrm{U} / 1$. Thus, those authors suggested that cirrhotic patients with cholinesterase of $<2,000 \mathrm{U} / 1$ may have higher risk for liver failure, if undergoing abdominal surgery. Thus, the combination cholinesterase of and the Child-Pugh score may be more subjective and accurate in evaluating the liver reserve function of cirrhotic patients, which is useful for surgeons to decide on surgery timing.

In conclusion, findings of the present study have demonstrated that the level of cholinesterase is closely correlated with the damage severity of liver cells and may respond to the liver reserve function of cirrhotic patients. Compared with the Child-Pugh score, serum cholinesterase is less complex and not easily affected by treatments for decompensated cirrhosis. The combination of cholinesterase with the Child-Pugh score may be more subjective and accurate in evaluating the liver reserve function of cirrhotic patients.

\section{Acknowledgements}

This study was supported by the National Natural Science Foundation of China (30901795).

\section{References}

1. Weisinger RA: Laboratory tests in liver disease and approach to the patient with abnormal test. In: Cecil Textbook of Medicine. Goldman L and Bennett JC (eds.). 21st edition, WB Saunders, Philadelphia, pp775-777, 2000.

2. Vincent D: Transaminases, or nithinecarbamyl tranferase, arginase, cholinesterase, etarylesterase. Lyon Med 232: 267-273, 1974 (In French).

3. Zhou X and Tu ZG (eds): Clinical Biological Chemical and Biological Chemical Inspection. 3rd edition. People's Medical Publishing House, Beijing, pp325-328, 2003.

4. Kariyone K, Shimatani Y, Kurihara T, Nagao T, Fujita Y and Uesugi M: Establishing indicators for diagnosis of cholinergic crisis. Rinsho Byori 58: 972-978, 2010 (In Japanese).
5. Lu Z and Zhong NS: Internal medicine. 7th edition. People's Medical Publishing House, Beijing, p453, 2008.

6. Zou Z, Xin S and Li B: Relationship between cholinesterase, prothrombin activity and albumin and the pathology of the liver. Zhonghua Shi Yan He Lin Chuang Bing Du Xue Za Zhi 15: 349-351, 2001 (In Chinese).

7. Association of Infectious and Parasitic Epidemiology Branch of Learning Branch of Liver Disease: viral hepatitis prevention and treatment plan. Chin J Inter Med 40: 62-68, 2001.

8. Sinha SN, Keresztes-Nagy S and Frankfater A: Studies on the distribution of cholinesterases: activity in the human and dog heart. Pediatr Res 10: 754-758, 2006.

9. Lampert IA and Van Noorden S: Acetyl cholinesterase is expressed in the follicular dendritic cells of germinal centres: differences between normal and neoplastic follicles. J Pathol 180: 169-174, 2006.

10. Kondo M, Hada T, Fukui T, et al: Enzyme-linked immunosorbent assay (ELISA) for aleuria aurantia lectin-reactive serum cholinesterase to differentiate liver cirrhosis and chronic hepatitis. Clin Chim Acta 243: 1-9, 1995.

11. Ogunkeye OO and Roluga AI: Serum cholinesterase activity helps to distinguish between liver disease and non-liver disease aberration in liver function tests. Pathophysiology 13: 91-93, 2006.

12. McArdle B: The serum cholinesterase in jaundice and diseases of the liver. Quart J Med 33: 107, 1940.

13. Wang J, Gao JX and Bai YZ: Patients with cirrhosis serum cholinesterase clinical research. Chin J Med Lab Sci 28: 68 , 2005.

14. Gu YJ and Zhong YB: Cholinesterase determination of evaluation of patients with liver cirrhosis of the liver reserves significance. Chin J Med Guide 12: 649-650, 2010.

15. Prakash R and Mullen KD: Mechanisms, diagnosis and management of hepatic encephalopathy. Nat Rev Gastroenterol Hepatol 7: 515-525, 2010.

16. Chromy V, Sváchová L, Novosád L, et al: Albumin-based or albumin-linked calibrators cause a positive bias in serum proteins assayed by the biuret method. Clin Chem Lab Med 47: 91-101, 2009.

17. Garg H, Kumar A, Garg V, Sharma P, Sharma BC and Sarin SK: Clinical profile and predictors of mortality in patients of acute-on-chronic liver failure. Dig Liver Dis 44: 166-171, 2012. 